ISSN 0103-9954

\title{
OCORRÊNCIA DE TÉRMITAS SUBTERRÂNEOS (ISOPTERA: RHINOTERMITIDAE E TERMITIDAE) E DURABILIDADE NATURAL DA MADEIRA DE CINCO ESSÊNCIAS FLORESTAIS
}

\author{
SUBTERRANEAN TERMITES (ISOPTERA: RHINOTERMITIDAE AND TERMITIDAE) OCURRENCE \\ AND WOOD NATURAL DURABILITY OF FIVE FOREST SPECIES \\ Henrique Trevisan $^{1}$ Janaína De Nadai ${ }^{1}$ Alexandre M. Lunz ${ }^{2}$ Acacio G. de Carvalho ${ }^{3}$

\section{RESUMO}

Avaliou-se a durabilidade natural da madeira de cinco essências arbóreas: Eucalyptus citriodora Hook, Gliricidia sepium (Jacq.) Steud, Lophantera lactescens Ducke, Piptadenia gonoacantha (Mart.) Macbr. e Samanea saman (Jacq.) Merr. O ensaio de campo foi realizado no campus da Universidade Federal Rural do Rio de Janeiro, de fevereiro de 2001 a novembro de 2002. Seis amostras de cada essência, parcialmente enterradas em posição vertical, foram dispostas aleatoriamente em cinco blocos. Em laboratório, segundo um sistema de notas relacionado ao estado fitossanitário da madeira, as amostras foram classificadas com um índice de comportamento, para cada essência, conforme seu aspecto fitossanitário. Também foram observados o estado da casca das amostras, a dureza da madeira e a presença de rachaduras longitudinais no corpo da amostra. As madeiras, mais e menos duráveis dentre os parâmetros avaliados, foram Laphantera lactescens e Piptadenia gonoacantha respectivamente. Em 47\% das amostras que foram atacadas, três espécies de térmitas foram identificadas: Coptotermes havilandi, Nasutitermes jaraguae e Nasutitermes minor.

Palavras-chave: durabilidade, ensaio de campo, Coptotermes, Nasutitermes.

\section{ABSTRACT}

This study aimed to evaluating of the wood natural durability of five arboreal essences: Eucalyptus citriodora Hook, Gliricidia sepium (Jacq.) Steud, Lophantera lactescens Ducke, Piptadenia gonoacantha (Mart.) Macbr. and Samanea saman (Jacq.) Merr. The field experiment was accomplished in the campus of the Universidade Federal Rural do Rio de Janeiro from February, 2001 to November, 2002. Six samples of each essence partially buried in vertical position were disposed at random in five blocks. In laboratory, following a system of notes related to the wood healthy state, the samples were classed accorging to a behavioral index for each species in relation to the wood aspect. The samples bark condition, the hardness of the wood and the presence of longitudinal cracks were also observed in the body of the sample Laphantera lactescens and Piptadenia gonoacantha were the most and less durable woods according to the evaluated parameters, respectively. In $47 \%$ of the samples that were attacked three species of termites were identified: Coptotermes havilandi, Nasutitermes jaraguae and Nasutitermes minor.

Key words: durability, field experiment, Coptotermes, Nasutitermes.

\section{INTRODUÇÃO}

A madeira tem sido utilizada pelo homem desde os tempos mais remotos e, mesmo com o surgimento de outros materiais que possam vir a substituí-la, sempre será interessante seu aproveitamento, seja pelos aspectos econômicos ou por suas características peculiares (Realino e Bueno, 1979).

A durabilidade natural da madeira permite avaliar a vida média útil e a susceptibilidade a organismos xilófagos. Desse modo, os resultados obtidos em ensaios de campo com madeiras em contato com o solo possibilitam a classificação da sua durabilidade, recomendando-se ou não o seu uso em ambientes externos (Jesus et al., 1998). Segundo Fosco Mucci et al. (1992), os ensaios de campo, embora

1. Acadêmicos do Curso de Graduação em Engenharia Florestal, Departamento de Produtos Florestais, Instituto de Florestas, Universidade Federal Rural do Rio de Janeiro. Rod. BR 465, km 7, CEP 23891-000, Seropédica (RJ). trevisan@ufrrj.br-janadenadai@bol.com.br

2. Engenheiro Florestal, Dr., Bolsista da CAPES, Instituto de Agronomia, Universidade Federal Rural do Rio de Janeiro. Rod. BR 465, km 7, CEP 23891-000, Seropédica (RJ). amehl@ig.com.br

3. Engenheiro Florestal, Dr., Professor Adjunto, Pesquisador do CNPq, Departamento de Produtos Florestais, Instituto de Florestas, Universidade Federal Rural do Rio de Janeiro. Rod. BR 465, km 7, CEP 23891-000, Seropédica (RJ). acacio@ufrrj.br

Recebido para publicação em 13/03/2003 e aceito em 3/09/2003. 
constituam métodos simples, reproduzem fielmente as condições reais de serviço nas quais peças de madeira de diferentes espécies e com dimensões preestabelecidas são expostas às intempéries e ao contato com o solo e avaliadas quanto ao grau de deterioração.

Dentre os organismos xilófagos existentes, destacam-se os insetos da ordem Isoptera, popularmente conhecidos como cupins ou térmitas, que constituem um grupo de reconhecida importância ecológica, pela ciclagem de nutrientes que promovem nos ecossistemas que habitam (Lelis, 1995). Contudo, são mundialmente reconhecidos pelos danos e prejuízos causados em certas culturas e em edificações e construções que empreguem quaisquer materiais de origem vegetal, pelas espécies que podem ser consideradas como pragas, que são poucas se comparadas às quase três mil espécies que compõem a ordem (Zorzenon e Potenza, 1998). Em estudos de degradação de madeira, os térmitas são classificados conforme o tipo de ninho que constróem, sendo dada ênfase aos cupins subterrâneos, tanto pela dificuldade no controle quanto pela proliferação maciça em ambientes urbanos (Santini, 1988). Atacam preferencialmente madeiras em contato com o solo, sendo facilmente perceptíveis pelas suas típicas galerias ascendentes.

Neste estudo, avaliou-se a durabilidade da madeira de cinco essências florestais visando à sua classificação com relação à durabilidade natural, baseada na intensidade do grau de deterioração e na susceptibilidade aos térmitas, bem como a identificação taxonômica destes.

\section{MATERIAL E MÉTODO}

\section{Caracterização do local}

A área de instalação do ensaio de campo está situada no campus da Universidade Federal Rural do Rio de Janeiro (UFRRJ), município de Seropédica, RJ ( $22^{0} 46^{\prime} \mathrm{S}, 43^{0} 41^{\prime} \mathrm{W}, 33 \mathrm{~m}$ ), com clima do tipo CWA, ou seja, quente e úmido (Köppen, 1948), com estação chuvosa no verão, e temperaturas bem distribuídas durante o ano todo (FIDERJ, 1978). A temperatura média anual é de $22,7^{0} \mathrm{C}$, e a precipitação anual varia em torno de $1200 \mathrm{~mm}$, segundo dados coletados na estação da Ecologia Agrícola da PESAGRO, no período de fevereiro de 2001 a novembro de 2002, quando da execução do estudo.

\section{Escolha e padronização das amostras}

As madeiras foram selecionadas aleatoriamente, com base na disponibilidade das cinco espécies no campus da UFRRJ, de regiões próximas à área escolhida: Eucalyptus citriodora Hook (Myrtaceae), Gliricidia sepium (Jacq.) Steud (Fabaceae), Lophantera lactescens Ducke, 'lanterneira' (Malpighiaceae), Piptadenia gonoacantha (Mart.) Macbr., 'pau-jacaré' (Mimosaceae) e Samanea saman (Jacq.) Merr. (Mimosaceae). De cada essência, foram utilizadas seis amostras, medindo 1,5 $\mathrm{m}$ de comprimento e entre 0,2 e $0,3 \mathrm{~m}$ de circunferência cada, com máxima uniformidade e sem defeitos na madeira, seguindo recomendações de Lopez e Milano (1986). Imediatamente após a coleta, elas foram dispostas na área experimental dividida em seis blocos, com cinco amostras por bloco. A disposição das amostras por essência em cada bloco foi aleatória, com espaçamento de 1,0 m entre amostras e 1,5 m entre blocos. As amostras foram enterradas verticalmente no solo a uma profundidade de $0,3 \mathrm{~m}$.

\section{Avaliação de parâmetros}

A única avaliação realizada no campo foi caracterizada com um leve movimento circular da amostra quando ainda no solo para verificar se a resistência havia sido reduzida de tal ponto a provocar a sua quebra. As demais avaliações foram feitas no Laboratório de Entomologia Florestal do Departamento de Produtos Florestais, Instituto de Florestas, UFRRJ, após 21 meses de exposição, onde o ataque de térmitas foi classificado segundo um sistema de notas relacionado ao estado fitossanitário da madeira (Tabela 1). Tal metodologia foi baseada em normas propostas por Lepage (1970) modificadas, para testes de campo com toras em contato com o solo. $\mathrm{O}$ índice de comportamento foi admitido para estabelecer o estado médio de cada amostra dentro de cada bloco; ao final, foram somados os índices de comportamento para cada essência, cujo valor foi dividido pelo número de amostras por essência (seis), obtendo-se o índice de comportamento médio. Também foram observados (1) o estado da casca das amostras, se aderida, solta ou ausente, (2) a dureza da madeira, mediante uma leve pressão com uma chave de fenda, e (3) a presença de rachaduras longitudinais no corpo da amostra. Os dois primeiros parâmetros foram avaliados tanto na parte

Ciência Florestal, v. 13, n. 2, 2003 
aérea quanto na parte subterrânea da amostra separadamente.

TABELA 1: Classificação do nível de degradação das madeiras em contato com o solo (Lepage, 1970).

TABLE 1: Classes of wood degradation level in contact with the soil (Lepage, 1970).

\begin{tabular}{l|c|c}
\hline Estado de Sanidade & Nota & Índice de Comportamento \\
\hline Sadio, nenhum ataque & 0 & 100 \\
Ataque leve ou superficial de fungos ou térmitas & 1 & 90 \\
Ataque evidente, mas moderado de fungos ou térmitas & 2 & 70 \\
Apodrecimento intenso ou ataque interno de térmitas & 3 & 40 \\
Quebra, perda quase-total de resistência & 4 & 0 \\
\hline
\end{tabular}

As espécies de térmitas coletadas nas amostras em laboratório foram etiquetadas e acondicionadas em álcool 70\% para posterior identificação no Departamento de Zoologia da Universidade de Brasília. Sinais de infestação de outros insetos xilófagos também foram observados.

\section{RESULTADOS E DISCUSSÃO}

Os maiores índices médios de comportamento foram verificados nas madeiras de Lophantera lactescens e Gliricidia sepium, com 87 e 72 respectivamente (Tabela 2). As amostras dessas essências foram as únicas a não terem sua resistência rompida pela força exercida na inspeção de campo e também as únicas a terem $100 \%$ de madeira dura na parte subterrânea da amostra. Tais índices médios para as amostras de Eucalyptus citriodora e Samanea saman foram de 40 e 33 respectivamente, sendo que em 50\% delas para cada essência foi observada madeira mole na parte subterrânea e quebra quando imprimido um leve esforço. Em todas as amostras de Piptadenia gonoacantha, o índice de comportamento foi mínimo (zero), com quebra mediante esforço e madeira mole na parte subterrânea.

TABELA 2: Nível de degradação das madeiras, expresso pelo índice de comportamento, pela percentagem de amostras com casca aderida, solta ou ausente, e percentagem de amostras com madeira dura, rachaduras longitudinais, ataque e espécies de térmitas incidentes.

TABLE 2: Wood degradation level expressed by the Behavioural index, for percentage of samples with attached, untied or absent bark, and for percentage of samples with hard wood, longitudinal cracks, attack and species of incident termites.

\begin{tabular}{|c|c|c|c|c|c|c|c|}
\hline \multirow[t]{2}{*}{ Madeira } & \multirow[t]{2}{*}{$\mathrm{IC}$} & \multicolumn{2}{|c|}{ Estado da Casca (\%) } & \multicolumn{2}{|c|}{ Dureza da Madeira (\%) } & \multirow{2}{*}{$\begin{array}{c}\mathrm{RL} \\
\text { (presença \%) }\end{array}$} & \multirow{2}{*}{$\begin{array}{c}\text { Ataque (\%) e } \\
\text { Espécie de Térmitas }\end{array}$} \\
\hline & & Solo (presença) & Parte Aérea & Solo & Parte Aérea & & \\
\hline $\begin{array}{l}\text { Lophantera } \\
\text { lactescens }\end{array}$ & 87 & 100 & $\begin{array}{l}17 \text { aderida } \\
33 \text { ausente } \\
50 \text { solta }\end{array}$ & 100 & 100 & 100 & $\begin{array}{l}17 \\
\text { Coptotermes } \\
\text { havilandi }\end{array}$ \\
\hline $\begin{array}{l}\text { Gliricidia } \\
\text { sepium }\end{array}$ & 72 & 16 & $\begin{array}{l}66 \text { aderida } \\
34 \text { solta }\end{array}$ & 100 & 100 & 50 & $\begin{array}{c}17 \\
\text { Nasutitermes minor }\end{array}$ \\
\hline $\begin{array}{l}\text { Eucalyptus } \\
\text { citriodora }\end{array}$ & 40 & 0 & $\begin{array}{l}33 \text { aderida } \\
33 \text { ausente } \\
33 \text { solta }\end{array}$ & 50 & 100 & 67 & $\begin{array}{l}\quad 67 \\
\text { Nasutitermes minor } \\
\text { Nasutitermes jaraguae }\end{array}$ \\
\hline $\begin{array}{l}\text { Samanea } \\
\text { saman }\end{array}$ & 33 & 50 & $\begin{array}{l}83 \text { aderida } \\
17 \text { solta }\end{array}$ & 50 & 100 & 17 & \begin{tabular}{l}
\multicolumn{1}{c}{33} \\
Nasutitermes minor \\
Coptotermes \\
havilandi
\end{tabular} \\
\hline $\begin{array}{l}\text { Piptadenia } \\
\text { gonoacantha }\end{array}$ & 0 & 67 & $\begin{array}{l}17 \text { aderida } \\
17 \text { ausente } \\
66 \text { solta }\end{array}$ & 0 & 100 & 33 & $\begin{array}{l}\qquad 100 \\
\qquad \begin{array}{l}\text { Nasutitermes minor } \\
\text { Coptotermes } \\
\text { havilandi }\end{array}\end{array}$ \\
\hline
\end{tabular}

Em que: $\mathrm{IC}=$ Índice de comportamento; $\mathrm{RL}=$ Rachaduras longitudinais.

A quebra nas amostras, cuja resistência foi afetada, ocorreu na região limítrofe entre o solo e a parte aérea. Embora cada grupo de espécies tenha registrado índices de comportamento diferenciados, variando de 0 a 87, em todas as madeiras, foi verificada consistência dura na parte aérea.

Rachaduras longitudinais ocorreram em todos as madeiras, todavia, em diferentes proporções. Em

Ciência Florestal, v. 13, n. 2, 2003 
todas as amostras de Lophantera lactescens, foi registrado esse parâmetro, seguido de Eucalyptus citriodora (67\%), Gliricidia sepium (50\%), Piptadenia gonoacantha (33\%) e Samanea saman (17\%) (Tabela 2). Esse comportamento diferenciado é relativo ao processo de secagem: as diferenças de umidade entre regiões contíguas de uma peça, nesse caso, entre a extremidade aérea exposta ao clima e a extremidade situada a $0,3 \mathrm{~m}$ de profundidade, condizem ao surgimento de tensões que levam à ruptura da madeira, quando superiores à resistência dos tecidos lenhosos (Galvão e Jankowsky, 1988).

Foi constatada a presença da casca no solo de todas as amostras de Lophantera lactescens, em 67\% das amostras de Piptadenia gonoacantha, 50\% de Samanea saman, 16\% de Gliricidia sepium e em nenhuma amostra de Eucalyptus citriodora. Os maiores valores encontrados para casca na parte aérea foram $83 \%$ de casca aderida nas amostras de Samanea saman, 66\% de casca solta em Piptadenia gonoacantha, e $33 \%$ de casca ausente em Lophantera lactescens e Eucalyptus citriodora (Tabela 2). A presença da casca no solo e o estado desta na parte aérea não devem ser considerados parâmetros isolados para classificação da durabilidade das madeiras, mas, sim, somados a outros aspectos que podem ajudar no entendimento da sua deterioração. De acordo com Burger e Richter (1991), a casca usualmente protege a madeira contra ressecamentos excessivos, ataques de organismos degradadores e variações climáticas. Isso pôde ser verificado nas amostras de Gliricidia sepium nas quais houve $66 \%$ de amostras com casca aderida na parte aérea, e em Lophantera lactescens, onde a casca esteve presente na parte subterrânea de todas as amostras. Contudo, nem sempre isso se verifica, podendo variar de espécie para espécie, como observado nas amostras de Samanea saman, em que nem os $83 \%$ de amostras com casca aderida na superfície foram suficientes para impedir um baixo índice de comportamento.

Foram encontradas três espécies de térmitas nas amostras avaliadas: Coptotermes havilandi Holmgren (Rhinotermitidae), e Nasutitermes jaraguae (Holmgren) e Nasutitermes minor (Holmgren) (Termitidae). Cerca de $47 \%$ das amostras foram atacadas, sendo que em todas as amostras de Piptadenia gonoacantha foram encontrados indivíduos de Coptotermes havilandi e Nasutitermes minor, as mesmas espécies de térmitas encontradas em 33\% das amostras de Samanea saman. Eucalyptus citriodora foi a segunda madeira mais atacada com $67 \%$ das amostras com presença de Nasutitermes minor e Nasutitermes jaraguae. Para a madeira de Lophantera lactescens e Gliricidia sepium, o ataque de térmitas ocorreu em $17 \%$ do material exposto, com ocorrência de Coptotermes havilandi e Nasutitermes minor respectivamente. Nasutitermes jaraguae ocorreu somente em amostras de Eucalyptus citriodora, Coptotermes havilandi foi coletado na madeira de Lophantera lactescens, Samanea saman e Piptadenia gonoacantha, e Nasutitermes minor ocorreu em todas as madeiras, exceto em Lophantera lactescens (Tabela 2). A resistência de muitas espécies florestais ao ataque de térmitas está relacionada à presença de substâncias químicas repelentes no lenho, normalmente tóxicas a esses insetos (Santini 1988) o que poderia explicar a menor incidência de ataque verificada na madeira de Lophantera lactescens e Gliricidia sepium. Fontes e Araújo (1999) destacam o gênero Coptotermes como o de maior interesse econômico nas áreas urbanas da região sudeste do País onde a espécie exótica Coptotermes havilandi é séria praga em casas, edifícios, construções, arborização urbana etc. Os mesmos autores também exaltam o gênero Nasutitermes como praga em diversas localidades do sudeste, leste e nordeste brasileiros.

Em cerca de $53 \%$ das amostras foram encontrados sinais da atuação de coleópteros das famílias Scolytidae, Cerambycidae e Bostrichidae, expressos na forma de galerias e orifícios de emergência que possibilitam a entrada de umidade na madeira, sendo sítios potenciais de desenvolvimento de fungos, parcialmente responsáveis pela aceleração da degradação do material. Aproximadamente $83 \%$ das amostras de Gliricidia sepium, Samanea saman e Piptadenia gonoacantha foram atacadas de forma substancial por essas coleobrocas, sendo que, para as duas primeiras, o ataque ocorreu de forma proporcional entre Scolytidae e Cerambycidae, enquanto que na última, o maior ataque foi feito por Cerambycidae e, em menor quantidade, por Bostrichidae. Para as madeiras de Eucalyptus citriodora e Lophantera lactescens, a atuação desses coleópteros resumiu-se em zero e 17\% respectivamente de amostras atacadas. Lunz e Carvalho (2002) ao avaliarem a degradação da madeira de Samanea saman, Eucalyptus citriodora e Lophantera lactescens causada por Scolytidae em contato com o solo por um período de oito meses registraram um maior número de perfurações para a primeira essência, seguida pela segunda e terceira, estas em número significativamente menor.

Ciência Florestal, v. 13, n. 2, 2003 
As informações obtidas neste estudo, juntamente com as propriedades tecnológicas dessas madeiras, complementarão o seu estudo, podendo fornecer subsídios para viabilização da comercialização e do uso correto da madeira dessas essências florestais. Além disso, tais informações auxiliam na compreensão do processo de degradação de madeiras existentes em nossos ecossistemas e na biologia dos térmitas que as degradam, dando mais subsídios para a intrincada relação desses degradadores com o complexo ambiente urbano em que vivemos. Novos trabalhos de análise da degradação de madeiras no campo são cada vez mais necessários e importantes para elucidar essa relação.

\section{CONCLUSÕES}

Das madeiras avaliadas, as mais duráveis, em termos de resistência, dureza e ataque de térmitas, quando em contato com o solo, são Lophantera lactescens e Gliricidia sepium, sendo seu uso indicado nesse ambiente.

A madeira de Piptadenia gonoacantha é de baixa resistência e altamente susceptível ao ataque de térmitas, conferindo a ela a menor durabilidade verificada dentre as madeiras testadas.

A durabilidade das madeiras de Eucalyptus citriodora e Samanea saman, no aspecto da resistência e dureza, é semelhante, podendo ser classificada como mediana.

Três espécies de térmitas foram coletados: Nasutitermes jaraguae ocorre somente em amostras de Eucalyptus citriodora; Coptotermes havilandi ataca a madeira de Lophantera lactescens, Samanea saman e Piptadenia gonoacantha, e Nasutitermes minor ocorre em todas as madeiras, exceto em Lophantera lactescens. Ao todo, $47 \%$ das amostras expostas foram atacadas por térmitas.

As madeiras de Lophantera lactescens e Eucalyptus citriodora tendem a rachar longitudinalmente com mais intensidade ao secarem em um período de tempo menor que as outras madeiras avaliadas.

Todas as madeiras das espécies testadas possuem resistência superior ao tempo de 21 meses de experimentação, referente ao aspecto dureza da madeira, no que tange ao uso fora do solo.

As madeiras de Gliricidia sepium e Samanea saman são susceptíveis ao ataque de Scolytidae e Cerambycidae (Coleoptera), Piptadenia gonoacantha é suscetível a Cerambycidae e Eucalyptus citriodora possui a madeira mais resistente dentre as testadas ao ataque de coleobrocas.

\section{AGRADECIMENTOS}

Ao prof. Dr. Reginaldo Constantino, do Departamento de Zoologia da Universidade de Brasília, pela pronta identificação dos cupins coletados nas amostras. Ao funcionário, da UFRRJ, Duclério José do Vale, por ceder espaço físico para montagem do experimento.

\section{REFERÊNCIAS BIBLIOGRÁFICAS}

BURGER, L. M.; RICHTER, H. G. Anatomia da madeira. São Paulo: Nobel, 1991. 154p.

FIDERJ. Indicadores climatológicos: sistema de informação para o planejamento estadual. Rio de Janeiro: SECPLAN, 1978. 156p.

FONTES, L. R.; ARAÚJO, R. L. Os cupins. In: MARICONI, F. A. M. (Coord.) Insetos e outros invasores de residências. Piracicaba: FEALQ, 1999. cap. 2, p. 35-90.

FOSCO MUCCI, E. S. C.; LOPEZ, G. A.; MONTAGNA, R. G. Durabilidade natural de madeiras em contato com o solo - IV. Revista do Instituto Florestal, São Paulo, v. 4, parte 2, p. 558-563, 1992.

GALVÃO, A. P. M.; JANKOWSKY, I. P. Secagem racional da madeira. São Paulo: Nobel, 1988. 112p.

JESUS, M. A.; MORAIS, J. W.; ABREU, R. L. S. et al. Durabilidade natural de 46 espécies de madeira amazônica em contato com o solo em ambiente florestal. Scientia Forestalis, Piracicaba, n. 54, p. 81-92, 1998.

KÖPPEN, W. Climatologia: com un estudio de los climas de la Terra. México: Fondo Cultural Económico, 1948. $479 \mathrm{p}$.

LELIS, A. T. Cupins urbanos: biologia e controle. In: BERTI FILHO, E.; FONTES, L. R. (eds.) Biologia e controle de cupins. Piracicaba: FEALQ, 1995. p. 77-80. 
LEPAGE, E. S. Método padrão sugerido pela IUFRO para ensaios de campo com estacas de madeira. Preservação de madeiras, São Paulo, 1970. v. 1, p. 205-216.

LOPEZ, G. A. C.; MILANO, S. Avaliação da durabilidade natural da madeira e de produtos usados na sua proteção. In: LEPAGE, E. S. (Coord.) Manual de preservação de madeiras. São Paulo: IPT-SICCT, 1986. v. 1, Cap. 10, p. 473521. (Publ. IPT, 1637).

LUNZ, A. M.; CARVALHO, A. G. Degradação da madeira de seis essências arbóreas disposta perpendicularmente ao solo causada por Scolytidae (Coleoptera). Neotropical Entomology, Londrina, v. 31, n. 3, p. 351-357, 2002.

REALINO, B. D.; BUENO, C. R. Durabilidade da madeira natural avaliada através de ensaios de campo. Revista Cientifica, Jaboticabal, v. 7, n. 1, p. 9-13, 1979.

SANTINI, E. J. Biodeterioração e preservação da madeira. Santa Maria: UFSM/CEPEF/FATEC, 1988. 125 p.

ZORZENON, F. J.; POTENZA, M. R. (Coords.) Cupins: pragas em áreas urbanas. São Paulo: Instituto Biológico, 1998. 46 p. (Boletim Técnico, 10). 